ENCYCIOPEDDE Encyclopédie berbère

BERBERE

28-29| 2008

28-29 | Kirtēsii - Lutte

\title{
Littérature berbère contemporaine
}

\section{A. Bounfour}

\section{OpenEdition}

Journals

Édition électronique

URL : http://journals.openedition.org/encyclopedieberbere/360

DOI : $10.4000 /$ encyclopedieberbere.360

ISSN : 2262-7197

\section{Éditeur}

Peeters Publishers

\section{Édition imprimée}

Date de publication : 1 janvier 2008

Pagination : 4435-4439

ISBN : 2-7449-0707-4

ISSN : 1015-7344

\section{Référence électronique}

A. Bounfour, "Littérature berbère contemporaine », Encyclopédie berbère [En ligne], 28-29 | 2008,

document L29b, mis en ligne le 01 juin 2013, consulté le 24 septembre 2020. URL : http://

journals.openedition.org/encyclopedieberbere/360 ; DOI : https://doi.org/10.4000/

encyclopedieberbere.360

Ce document a été généré automatiquement le 24 septembre 2020.

(c) Tous droits réservés 


\section{Littérature berbère contemporaine}

\section{A. Bounfour}

1 La production littéraire d'aujourd'hui jouit de plusieurs dénominations : «littérature berbère contemporaine ", « littérature moderne » ou "néo-littérature ». Chacune de ces trois appellations pose des problèmes spécifiques. La première risque de faire croire que l'on dispose d'une histoire de la littérature scandée en périodes, ce qui, hélas, n'est pas le cas; la seconde présuppose que la tradition est close par un concept moderne isomorphe à celui du monde occidental, ce qui est faux ; et la troisième présuppose qu'il existait un régime clos de la littérature aujourd'hui dépassé, ce qui est inexact.

2 Ceci étant, on peut adopter une de ces dénominations en en explicitant le sens. On utilisera ici la première car elle est la plus simple à expliciter : il suffit de rappeler que ce sont les textes produits aujourd'hui (nos contemporains et non la contemporanéité au sens historique en Europe) et véhiculés par l'écrit et encadrés par un droit régissant cet écrit. Une telle définition nous introduit directement aux problèmes majeurs de cette littérature dont son rapport au régime de production littéraire traditionnel. De ce point de vue, elle est en rupture plus ou moins radicale avec ce régime.

\section{Oralité-vocalité et écriture}

3 Le berbère est toujours décrit comme langue orale ou "essentiellement orale ». La seconde formulation est la plus adéquate car cette langue a connu l'écriture depuis fort longtemps. Ce rapport mérite attention. On se limitera ici à la tradition manuscrite berbère en caractères arabes.

4 Les textes littéraires transmis sont essentiellement religieux, mystiques ou moraux. Leur mise en écriture a pour fonction leur conservation et leur transmission pour qu'ils soient récités ou cantilés, souvent par des groupes de disciples d'un soufi, ou par la communauté villageoise célébrant un rituel ou une fête religieuse ou même " profane ». L'écriture est donc essentiellement un moyen de transcription et de conservation des textes. Leur consommation nécessite obligatoirement leur actualisation par la voix. C'est pourquoi on est fondé de parler du caractère fondamentalement vocal de cette littérature même si elle est écrite. Il faudrait plutôt dire qu'elle est transcrite. 
5 La littérature contemporaine a un rapport plus complexe avec l'écriture. On y trouve des textes dont la typologie va de la vocalité traditionnellejjusqu'à l'inter textualité scripturaire la plus éloignée de cette vocalité ${ }^{2}$.

\section{Performance in situ et performance décalée}

6 La performance des textes vocaux a des contraintes spécifiques : un exécutant, un public disponible et une temporalité déterminée. Le texte n'existe que dans cette situation dans laquelle il est œuvre esthétiquement recevable. Et chaque fois qu'il est actualisé ainsi, chacune de ses actualisations est une œuvre à part entière. Il l'est pour l'exécutant et pour le public. Ce mode de réception montre bien que le texte n'est pas l'essentiel de l'œuvre ; c'est son actualisation vocale dans une situation déterminée qui l'est. Le texte existe in situ ou n'existe pas.

7 En revanche, le texte contemporain existe par lui-même porté par le nom d'un auteur et non d'un exécutant. Il est reçu par un lecteur solitaire et n'a pas besoin d'être dit en présence d'un public sauf dans ce qu'on appelle des lectures qui sont des situations plus promotionnelles que des réceptions formalisées des textes ${ }^{3}$.

\section{La littérarité}

8 Si la littérarité du texte traditionnel est fondée sur la satisfaction de l'attente de l'auditeur, il va sans dire que le plaisir littéraire a pour socle le convenu dont les figures fondamentales sont le cliché et la répétition. Le bon poète, par exemple, ne doit pas surprendre son auditeur par des mots recherchés, des métaphores inédites, des idées neuves. Tout cela participe de l'obscurité du texte alors qu'on attend de ce dernier qu'il soit compris dans l'immédiateté de son dire. L'esthétique traditionnelle répugne à l'effort d'interprétation : tout texte non saisi au fur et à mesure de son déploiement est alors mal conçu.

9 Tel n'est pas la littérarité contemporaine. Sans rompre totalement avec la précédente dont elle joue, elle se fonde sur la représentation de la création artistique comme quelque chose d'inédit, représentation bien romantique, certes, mais réelle.

10 Le poète crée du nouveau dans la thématique, la stylistique et même dans la langue. En effet, la littérature contemporaine foisonne de néologismes. Pas un texte sans un lexique qui l'accompagne pour saisir le sens de certains mots, de certaines locutions. Pas un texte sans nouvelles comparaisons, sans métaphores insolites, surprenantes car jamais usitées dans la langue « traditionnelle », y compris la langue poétique.

11 Sur le plan thématique, on abandonne les contenus convenus de la tradition pour introduire des thèmes inspirés de la réalité sociale et politique. C'est ainsi que le thème de l'identité berbère est omniprésent, voire obsédant. Même le thème de la passion amoureuse, si convenu et si universel, est traité autrement, à la lumière de la vie d'aujourd'hui et non selon des canons immuables de la tradition. La religion elle-même, malgré sa sacralité renforcée par l'ordre juridique des États nationaux, n'échappe pas à un traitement au moins irrespectueux, voire iconoclaste (voir Id Belqacem). 


\section{Le système générique}

12 À ce changement thématique correspond une véritable substitution de système générique pour autant que l'on puisse parler de système. On distinguera le système oral de celui de l'écrit.

En effet, les grands genres traditionnels oraux sont le conte sous toutes ses formes, le proverbe, l'énigme et la devinette. L'essentiel de la littérature narrative passe par le conte merveilleux, la fable, l'anecdote plaisante. Cette narration est souvent en prose, mais tout un pan est en vers. Poésie et prose sont des modes d'expression utilisés par tous les genres et tous les types. C'est ainsi que les jeux de langage dont le proverbe et l'énigme peuvent, eux aussi, être en vers ou en prose.

litterarité contemporaine, à l'instar d'autres littératures anciennes ou récentes, a tout simplement adopté les genres occidentaux : roman, nouvelle, théâtre, etc.

Même si la poésie reste le domaine le plus productif, le roman est le genre le plus significatif de cette littérature pour plusieurs raisons :

Le roman confronte le romancier à l'invention véritable non seulement d'un bien dire, mais de tout un univers de paysages, d'atmosphères, de personnages différenciés dans leurs comportements y compris dans leurs langages. De plus, le romancier n'a pas de modèle autochtone sur qui s'appuyer. C'est cette difficulté qui explique, entre autres, que les premiers textes narratifs berbères soient inspirés de contes ou des tentatives de courts récits plus proches de la nouvelle que du roman. D'ailleurs, tous les textes publiés à ce jour comme roman sont, dans le meilleur des cas, des nouvelles plus ou moins développées.

Le roman devient le lieu où s'expriment les désirs, les fantasmes et les frustrations profondes des nouvelles générations, expression qui n'a nul autre lieu pour venir au monde étant donné que le système traditionnel ne peut exprimer que des convenances. Le roman et la nouvelle sont les lieux où l'amour, par exemple, la politique, la religion, c'est-à-dire la trinité du tabou des sociétés berbères d'aujourd'hui, sont traités avec une liberté en rupture avec la tradition qui, généralement, utilise la suggestion la plus vague quand elle ose affronter ces problèmes.

17 Cette thématique impose au romancier et nouvelliste une attention accrue à sa propre expérience et à celle de sa société. C'est pourquoi l'aspect réaliste de cette littérature est frappant. Il faut le considérer comme une nouveauté et, par conséquent, comme élément de l'esthétique contemporaine. Le poète traditionnel n'ignorait pas sa société et sa propre expérience. Le mode d'expression n'est pas le réalisme, mais un symbolisme convenu, contrôlé et maîtrisés. Or, le réalisme permet une liberté d'expression pour autant que le romancier n'est pas assujetti à un modèle réaliste, sinon le sien propre avec son propre langage.

Le roman est aussi un lieu de renouvellement de la langue. Certes, on peut regretter des abus dus souvent à des ignorances, mais aucune littérature n'a échappé à cet écueil. Il suffit de rappeler l'expérience de La Pléiade française et le mouvement étymologiste qui l'avait accompagné.

La poésie reste encore la reine de la production littéraire berbère d'autant plus qu'elle bénéficie des moyens audio-visuels modernes grâce à sa mise en musique et aux chanteurs. De ce point de vue, elle est le mode d'expression qui a contribué de manière remarquable à l'émergence politique et médiatique de l'identité berbère et lui a donné 
une dimension internationale. Les noms des grands poètes baladins ne manquent pas : Idir, Aît Menguellat et Matoub, entre autres, en Kabylie ; le groupe Ousmane et Amori en pays chleuh qui ont chanté des poèmes écrits par divers poètes, etc.

La contemporanéité de cette poésie réside essentiellement dans sa thématique renouvelée dont le thème de l'identité berbère est lancinant: Qui sommes-nous? Pourquoi sommes-nous dominés? Qui est responsable de cet état de fait? À ces questions les réponses sont données par une relecture de l'histoire ancienne et/ou récente, par la sociologie, etc. On dira, sans hésitation, que la poésie chantée a popularisé les idées que les intellectuels berbères, leurs créateurs, n'ont pu porter au-delà de leurs cercles.

\section{L'édition}

Il n'est pas question de donner une liste exhaustive de la production littéraire berbère contemporaine, mais d'indiquer les références essentielles où l'on peut trouver les titres de ces œuvres.

En effet, l'édition de ces œuvres est si variée et dispersée que l'on peut véritablement s'y perdre. Il n'existe pas d'éditeur spécialisé dans ce domaine dans les pays berbérophones. De plus les législations sont si diverses qu'il est difficile de s'y retrouver. C'est ainsi, par exemple, qu'au Maroc un écrivain peut publier ses textes chez un éditeur ou à compte d'auteur et s'instituer ainsi son propre éditeur, voire son propre distributeur. D'ailleurs, c'est cette dernière solution qui a permis la publication de la plupart des textes littéraires ou autres berbères. Cette option semble plus récente en Algérie où il y avait des institutions étatiques de publication comme l'ancienne SNED, par exemple. Aujourd'hui un romancier talentueux comme Mezdad peut publier à compte d'auteur.

Le revers de la médaille de cette formule n'est pas à sous-estimer: des textes commencent à être publiés dans des bourgs coupés de tout réseau de communication par des associations ou des individus. Seul le hasard ou la curiosité du voyageur peut porter à la connaissance des amateurs cette production.

Toutefois, la bibliographie berbère tenue à jour par $\mathrm{S}$. Chaker durant une décennie puis par S. Chaker et A. Bounfour a recensé les œuvres parues durant cette période. On peut s'informer aujourd'hui auprès du Centre de Recherche Berbère de l'INALCO, d'un éditeur-libraire berbère parisien spécialisé dans le domaine du berbère.

\section{Conclusion}

Cette présentation rapide de la littérature berbère contemporaine ne manquera pas de faire penser à la dynamique des autres littératures africaines ou autres qui émergent ou se consolident dans les langues autochtones.

Cette dynamique semble "universelle » et mériterait que les spécialistes de ces littératures initient un comparatisme qui permettrait de cerner comment émerge et se développe une littérature, comment se constitue une esthétique, une langue littéraire. Ceci est d'autant plus urgent que des corpus se constituent sous nos yeux. 


\section{BIBLIOGRAPHIE}

Voir : « La littérature berbère contemporaine », dossier coordonné par S. CHAKER \& A.

BOUNFOUR, dans la revue Études Littératures Africaines (Bordeaux), 21, 2006 (6 contributions).

BRENIER-ESTRINE Cl., Bibliographie berbère annotée 1993-1994, CNRS/IREMAM, Aix-Marseille, 1995.

CHAKER S., Une décennie d'études berbères (1980-1990). Bibliographie critique, Bouchène, Alger, s.d.

CHAKER S. (avec la collaboration de) A. BOUNFOUR, Langue et littérature berbères. Chronique des études XII (1992-1993), INALCO, Paris, 1994.

CHAKER S. et BOUNFOUR A., Langues et littératures berbères. Chronique des études XIII (1994-1995), L'Harmattan, Paris, 1996.

GALAND L., Langue et littérature berbères. Vingt cinq ans d'études, Editions du CNRS, Paris, 1979.

Voir aussi la bibliographie berbère, interrogeable en ligne, sur le site Internet du Centre de Recherche Berbère : http://www.inalco.fr/crb/.

\section{NOTES}

1. Un exemple évident de poésie vocale transcrite: Jouhadi Houssein, Timatarin (Avertissements), Casablanca, 1997.

2. Un exemple où la vocalité est plus dans la versification que dans les métaphores: Ali Azaykou, Timitar (Signes), okad, Rabat, 1988.

3. Les textes de théâtre sont, évidemment, à excepter de cette assertion.

\section{INDEX}

Mots-clés : Culture, Poésie, Roman 